\title{
HPV co-testing as cervical screening test. Experience of Alweiya early detection cervical clinic: one-year analysis
}

\author{
Zainab J Al-Jobawi' ${ }^{1}$, Besmah M. Ali², Asan A Al-Niyazee³, Mustafa H Ibraheem³
}

1 Cancer screening fellowship, Babil Health Directorate, Ministry of Health, Iraq.
2 Gazi Al Hariri hospital, Ministry of Health, Baghdad, Iraq.
3 AL-Alwiyaa maternity teaching hospital, Ministry of Health, Baghdad, Iraq.
Correspondence: Dr.Zainab J. Al-Jobawi (E-mail: zainabjalil5@gmail.com)
(Submitted: 18 July 2020 - Revised version received:26 July 2020 - Accepted: 19 August 2020 - Published online: 30 October 2020)

\begin{abstract}
Objectives: The high-risk human papilloma virus test with conventional cytology (HPVco-test) was firstly introduced in our institute in May 2019. So, we aimed to estimate the prevalence of abnormal cytology and positive high-risk human papillomavirus test results in a screening woman and to assess the accuracy between cytology and human papilloma virus testing to evaluate the feasibility of integrating the latter as a primary test in the national cervical cancer screening program.

Methods: A prospective study for women attending to early detection cervical clinic, during the period from May 2019 to May 2020. Patients who were sexually active were included in the study. Samples for conventional cytology and HPV by using real-time polymerase chain reaction technique for high risk types were taken concurrently. The prevalence of positive screening results and the difference in accuracy between two testing were estimated by McNemar's $x^{2}$ test.

Result: A total of 388 women were included in the study. The prevalence of positive test for hr-HPV was 2.1\% (8) while the prevalence of abnormal cytology test was $19.1 \%$ (74). Concerning discordant pairs, $0.8 \%$ (3) of women had normal cytology with a positive hr-HPV test result and $17.8 \%$ (69) of women had abnormal cytology with a negative hr-HPV test result. A total of 311 (80.1\%) women had normal cytology and negative hr-HPV test results. The proportion of women with abnormal cytology and positive hr-HPV test results was $1.3 \%$ (5 women). The difference in accuracy between the two results was statistically significant $(<0.0001)$. The prevalence of positive $h r-H P V$ test decreased with increasing age, whereas the prevalence of abnormal cytology showed a bimodal age pattern.

Conclusion: The prevalence of abnormal cytology was high to that of hr-HPV testing, which could not allow for the implementation of hrHPV as a primary test in the national screening program in Iraq.

Keywords: Conventional cytology, hr-HPV, HPV co-test, Abnormal cytology, Cervical cancer.
\end{abstract}

\section{Introduction}

Cervical cancer is ranked as the fourth most frequently diagnosed cancer and the fourth leading cause of cancer death in women worldwide. Cervical cancer continues to be a major public health problem affecting middle-aged women in 42 low-resource countries. ${ }^{1}$ In Iraq, cervical cancer is 12 th cancer among women, and the 10th among those aged between 15 and 44 years old. ${ }^{2}$ Virtually, the cervical precancerous changes occur mainly due to the infection with human papillomavirus (HPV), which has many oncogenic subtypes, including: $16,18,31,33,35,39,45,51,52$, 56,58 , and 59 which is classified as Group 1 carcinogens by the IARC Monographs. ${ }^{3}$ In spite of the majority of HPV infections are curable spontaneously and don't lead to precancerous cell changes, but the persistent infection with carcinogenic HPV types is the main cause in triggering the development of cervical cancer. ${ }^{4}$ Other important cofactors include immunosuppression (particularly human immunodeficiency virus), smoking, parity (a higher number of full-term pregnancies increases risk), and oral contraceptive might also contribute to precancerous changes. ${ }^{5}$

Prevention, early detection, control, and treatment of the pre-invasive uterine cervical lesions are essential methods to limit cervical cancers and are imperative ways in decreasing early death throughout the productive period in a woman's life. Early detection occurs through screening test, which include cytology, HPV testing, and VIA. Screening through cytology has undoubtedly led to a major decline in cervical cancer burden in several resource-rich countries, however, the method might have reached its limits, with reports from several countries with longstanding high-quality cytology-based programmers indicating that trends have either stabilized or began to rise. ${ }^{6}$ Meta-analyses and pooled analyses of randomized trials have shown that screening with HPV tests protects better against future cervical precancerous lesions and invasive cancers than screening by cytology ${ }^{7-9}$ and, therefore, virological screening programmes are becoming increasingly recommended. ${ }^{10,11}$ Routine cervical screening of women 30 years and older using Papanicolaou (Pap) cytology and HPV together (co-testing) was first approved by the US Food and Drug Administration in 2003. ${ }^{12}$ Currently, there are two different major guidelines for hr-HPV testing. In the USA, combined hr-HPV and cytology testing every 5 years is an alternative to cytology testing every 3 years. ${ }^{13}$ In Europe, co-testing is discouraged at any age, and European guidelines recommend hr-HPV as a primary screening test for women above 30 years of age, owing to convincing evidence for more efficacious screening. ${ }^{14}$ Early detection in our institute till May 2019 was done by primary cytology test, visual acetate acid inspection (IVA) inspection method. After that, hr HPV co testing was established as a screening test and as hr-HPV testing is not offered in the public health sector in Iraq, and there are no data available on HPV prevalence in the general, asymptomatic female population. So, we aimed to estimate the prevalence of abnormal cytology and positive hr-HPV test results in a screened woman and to evaluate the feasibility of integrating the hr-HPV co-testing as a primary test in the national cervical cancer screening program. 


\section{Patients and Methods}

A prospective study for women attending to Alweiya early detection cervical clinic, which belongs to alrosafa health directorate, Baghdad, Iraq, during the period from May 2019 to May 2020. These women either suffered from genital health problems or just attended the aforementioned center for screening. All patients were aware of cytological examination and its purpose. Patients who were sexually active were included in the study. Samples for conventional cytology and hr-HPV testing were taken concurrently. Samples were obtained by rotating a SPLCyto Pap Brush (SPL Life Sciences, Pocheon, South Korea) five full turns in the cervical region. Cytology was performed at the reference laboratory of the AL-Alwiyaa maternity teaching hospital. Samples were analyzed by one cytopathologist. Cytological results were classified based on the 2001 Bethesda system into, ${ }^{15}$ where the smears that interpreted negative for intraepithelial lesion, were considered normal cytology result and the smears that interpreted as atypical squamous cells of undetermined significance (ASC-US, possibly nonneoplastic); atypical squamous cells (ASC-H, cannot exclude HSIL); atypical glandular cells (AGC); low-grade squamous intraepithelial lesion (LSIL); high-grade squamous intraepithelial lesion (HSIL); invasive squamous cell carcinoma (SCC); adenocarcinoma in situ (AIS); invasive adenocarcinoma and invasive endometrial adenocarcinoma, were considered as abnormal cytology result. Hr-HPV testing was done at the Central Public Health Laboratory, Molecular Biology Unit, to detect HPV DNA in cervical swabs by using real-time polymerase chain reaction (PCR) technique for high risk types (16, $18,31,33,35,39,45,51,52,56,58,59)$. All samples were blinded to cytology results. The data obtained were tabulated in a Microsoft Excel spreadsheet and subsequently exported to the SPSS Statistics software package, version 23. Descriptive data on the study population are presented in absolute and relative frequencies. Continuous variables are presented as mean $\pm \mathrm{SD}$. The overall prevalence of positive cytology and hr-HPV test result and by age was reported. Differences in positive hr-HPV test result by type of abnormal cytology result were tested with $\mathrm{McNemar}$ 's $\chi^{2}$ test. $P$ values less than 0.05 were considered statistically significant.

\section{Result}

A total of 388 women were enrolled in this study, the mean age (SD) of women was 40.4 (10.1) years, age range was between 23 and 65 years. From all women, only 40 (10.3\%) women was came for screening, other women were referral cases (Table 1). The prevalence of positive test for hr-HPV was $2.1 \%$ (8), while the prevalence of abnormal cytology test was $19.1 \%$ (74), Concerning discordant pairs, $0.8 \%$ (3) of women had normal cytology with a positive hr-HPV test result and $17.8 \%$ (69) of women had abnormal cytology with a negative hr-HPV test result. A total of 311 (80.1\%) women had normal cytology and negative hr-HPV test results. The proportion of women with abnormal cytology and positive hr-HPV test results was $1.3 \%$ (5 women). The difference in accuracy between the two results was statistically significant $(p=<0.0001$ ) (Table 2 )

The pattern of cytological abnormality shown by cytology was, 33.8\% (25) of participants had atypical squamous cell of undetermined significant (ASCUS), 58.1\% (43) of women had low squamous intraepithelial lesion (LSIL), 4\% (3) of women

\begin{tabular}{|c|c|c|c|}
\hline \multicolumn{2}{|c|}{ Characteristic } & Number & Percentage \\
\hline \multirow{5}{*}{ Age } & $<30$ years & 62 & $16 \%$ \\
\hline & 30-39 years & 121 & $31.2 \%$ \\
\hline & 40-49 years & 128 & $33 \%$ \\
\hline & 50-59 years & 62 & $16 \%$ \\
\hline & $\geq 60$ years & 15 & $3.9 \%$ \\
\hline \multirow{2}{*}{$\begin{array}{l}\text { Mode of } \\
\text { detection }\end{array}$} & Screening & 40 & $10.3 \%$ \\
\hline & Symptomatic & 348 & $89.7 \%$ \\
\hline
\end{tabular}

Table 2. Concordance of cytology and hr HPV test in HPV co testing.

\begin{tabular}{cccccc}
\hline $\begin{array}{c}\text { Result of HPV co } \\
\text { test }\end{array}$ & \multicolumn{2}{c}{ Cytology test } & Total & Pvalue \\
& Abnormal & Normal & & \\
Hr HPV Positive & 5 & 3 & $8(2.1 \%)$ & \\
& Negative & 69 & 311 & $380(97.9 \%)$ & $<0.0001$ \\
Total & $74(19.1 \%)$ & $314(80.9 \%)$ & 388 & \\
\hline
\end{tabular}

McNemar's $\times 2$ test, significant $\leq 0.05$.

had high squamous intraepithelial lesion (HSIL), 2.7\% (2) of women had atypical glandular cell (AGS), and only 1 woman had ca. cervix (Fig 1).

The prevalence of positive hr-HPV test result varied considerably with age, the prevalence decreased from $3.2 \%$ in women aged $<30$ years to $2.5 \%$ in women aged $30-39$ years, and $1.6 \%$ in women aged $40-59$ years (Fig 2).

There was a slight increase in the prevalence of abnormal cytology test among women $30-39$ years and $50-59$ years compared with women aged $<30$ years and $40-49$ years (Fig 3 ).

\section{Discussion}

This is the first study in Iraq to assess the prevalence of abnormal cytology and positive hr-HPV test results using hr-HPV co test. We found that among 388 women with acceptable results for both tests, the population prevalence of abnormal cytology results was $19.1 \%$ and the prevalence of positive hr-HPV was $2.1 \%$ and the difference in accuracy between the two results was statistically significant. We found a higher prevalence of abnormal cytology than found in populations tested on a regular basis such as in Romania and Turkey (the $12-11.5 \%$. respectively). ${ }^{16,17}$ This could be explained by the prevalent and incident cases discovered in our sample, in contrast to only incident cases found in regularly tested women found in that studies and also in our sample, majority of women were symptomatic (89.7\%) and the frequency of abnormal cytological result in symptomatic women was higher and this proved by many study. ${ }^{18,19}$

The prevalence of abnormal cytology result showed a bimodal age pattern result which not in line to that reported in some other populations, where shown increased in prevalence of abnormal cytology with increasing age. ${ }^{20,21}$ Other studies showed a reversed result where the prevalence of abnormal cytology decrease by increase in age. ${ }^{22,}{ }^{23}$ Unfortunately, in Iraq there 


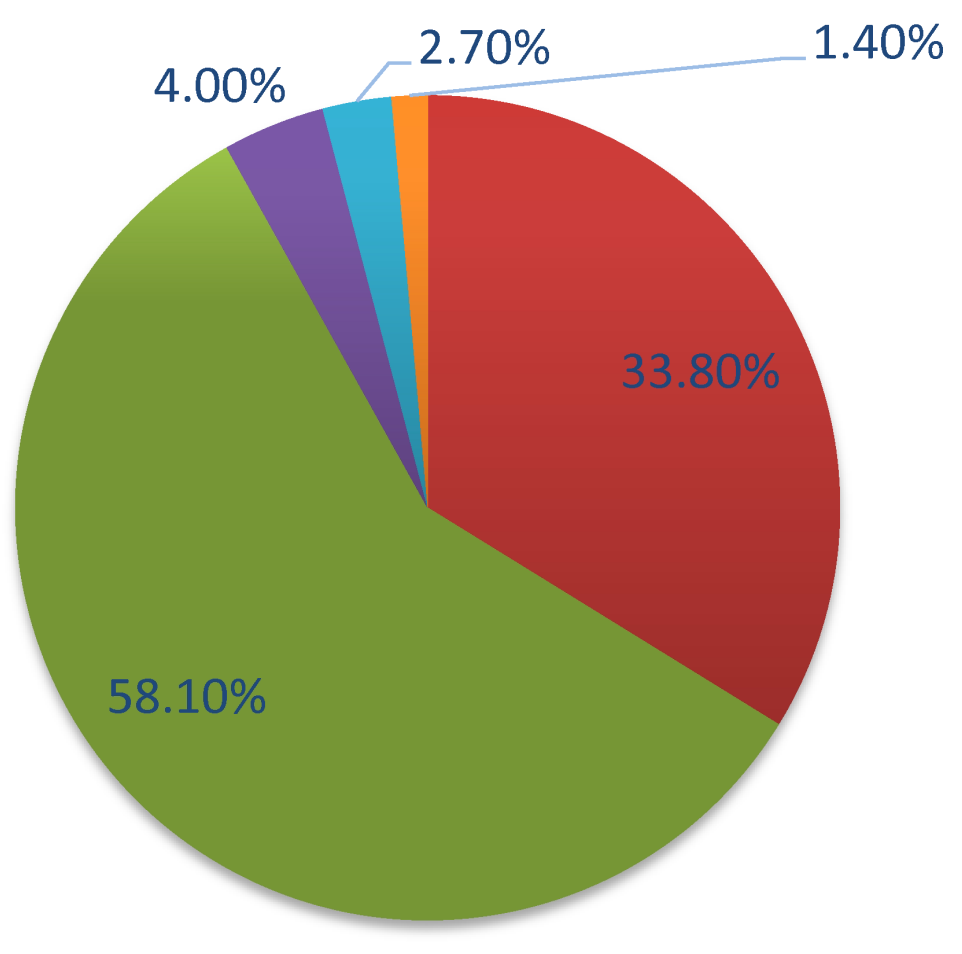

Fig 1. The pattern of cytological abnormality shown by cytology.

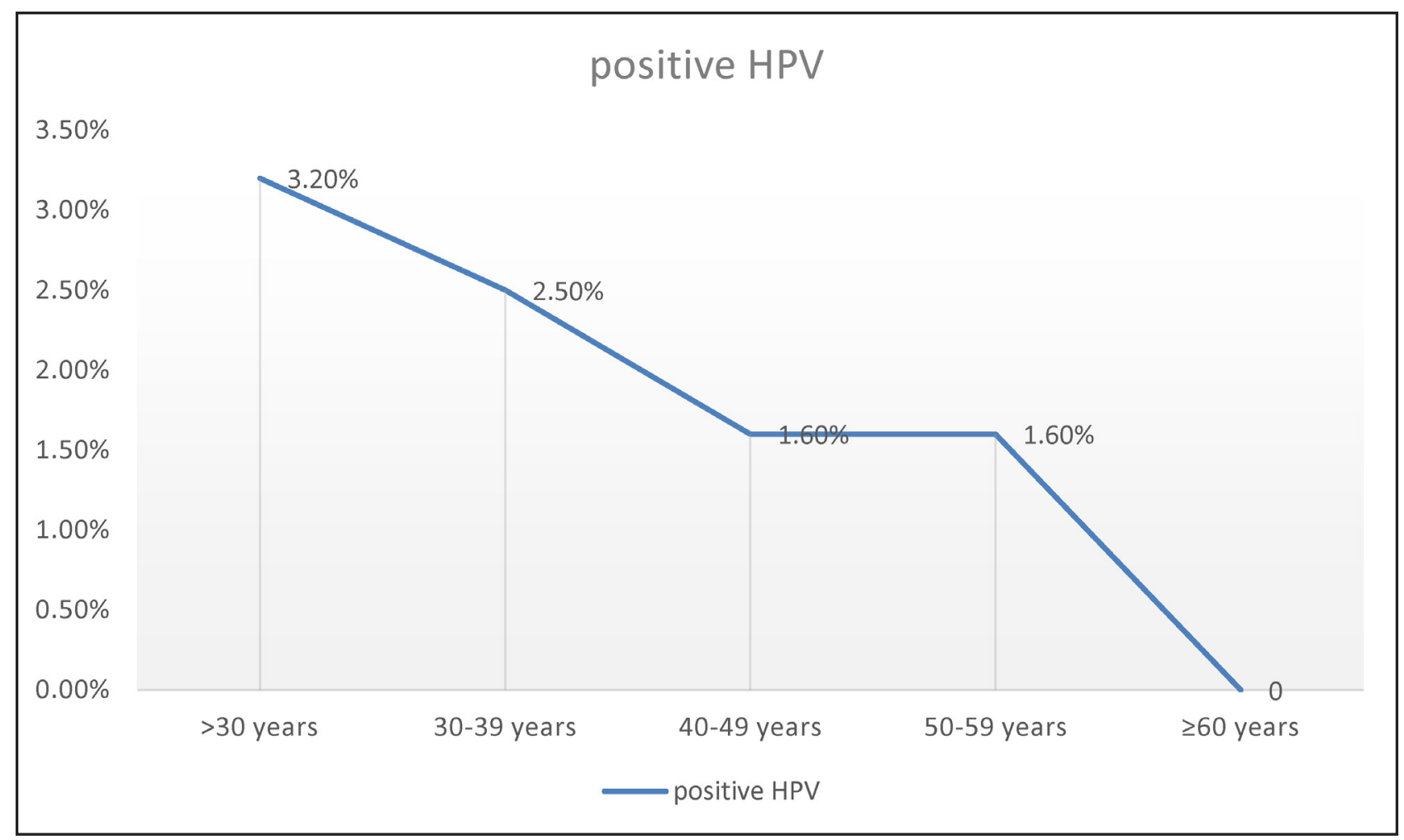

Fig 2. Prevalence of positive hr-HPV infection result by 10-year age group. 


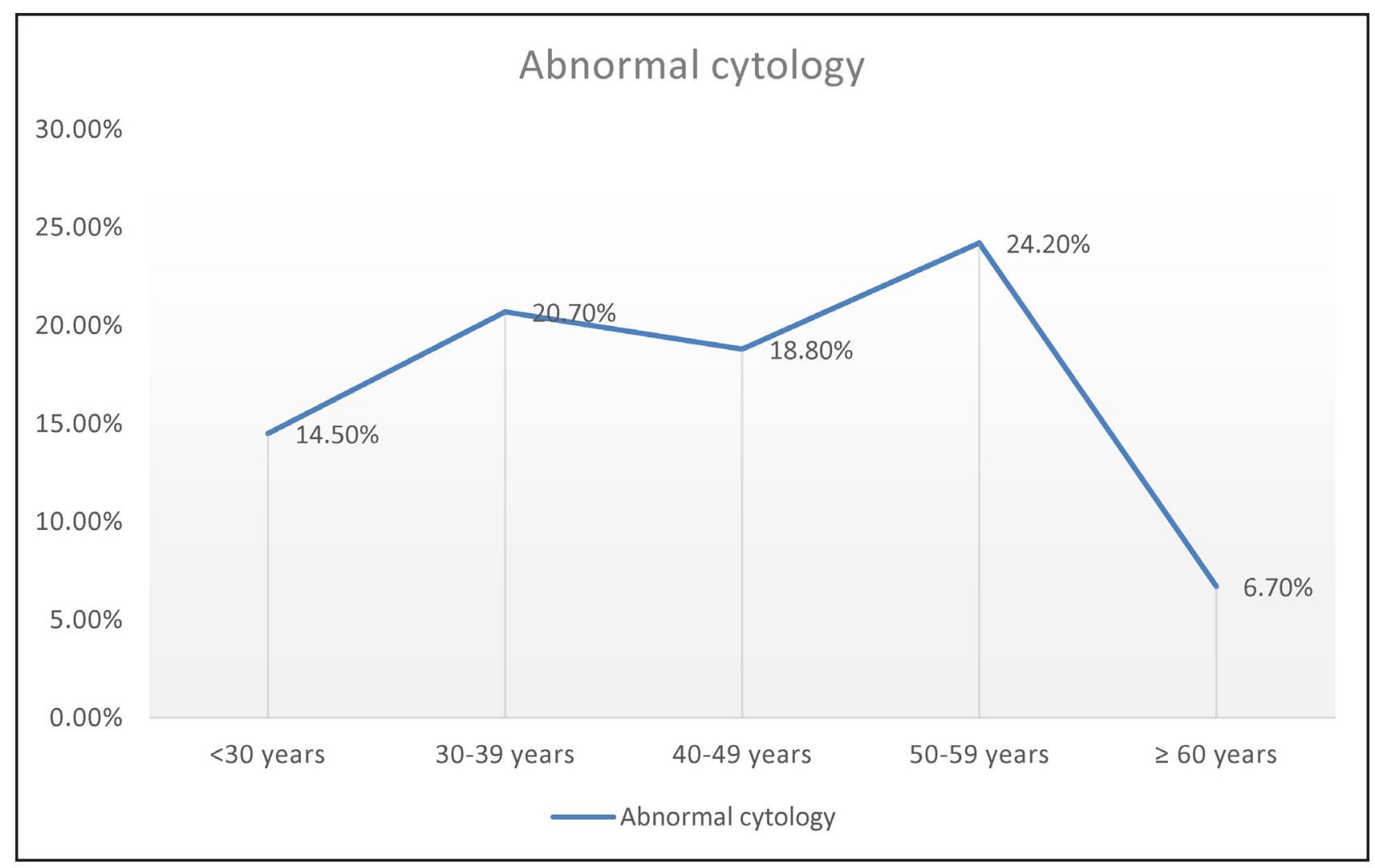

\section{Fig 3. Prevalence of abnormal cytology by 10 -year age group.}

were no population-based study which investigated the prevalence of HPV in women, In this study, the prevalence of positive hr-HPV was low compared to other population. The most plausibly explanation related to societal factors and sexual behavior, also our country exhibit low rates of other sexually related infections, such as HIV. ${ }^{24}$ In our study, the peaks in hr-HPV prevalence was in women aged less than 30 years and the prevalence decrease by increase age. This explained that younger age had a high proportion of positive results which reflects infections that will clear spontaneously (transient hr-HPV infections that eliminated by the immune system in 6-18 months) without reflected in cellular atypia and this in line of other studies. ${ }^{23,25}$

Interestingly, we found that the likelihood of hr-HPV positive testing but cytologic negative to be a less common than the likelihood of hr-HPV negative testing but cytologic positive ( $0.8 \%$ vs. $17.8 \%)$, The $0.8 \%$ of women with normal cytology and positive hr-HPV results was lower to the percentage obtained in the ARTISTIC trial (9\%), ${ }^{26}$ lower than worldwide estimate of $11.7 \%,{ }^{27}$ and substantially lower than previously reported percentages from Eastern Europe ${ }^{28,29} 14.0 \%$ in southeastern Asia to $14.4 \%$ in south central Asia. ${ }^{30}$ Variations between studies most likely reflect differences in the population studied with respect to risk factors for exposure to HPV and methods of evaluations. The $17.7 \%$ of women with negative hr-HPV test results and abnormal cytology, where the cytological abnormality in our study where primarily ASC-US cytology and LSIL and those abnormality harbor low prevalence of HPV infection by many studies, ${ }^{31,32}$ also those abnormality bears a low risk of CIN $2 / 3 .{ }^{33}$ These women require surveillance and follow-up according to guidelines, as they have an elevated risk for cervical lesions over time. ${ }^{34}$
Recognition of $80.2 \%$ of women with cytologically normal and hr-HPV-negative women is important because extension of screening intervals from 3 years based on negative cytology alone to 5 years based on hr-HPV co test.

The present study has several limitations, including the use of data from a single provider of health-care services and not based on the Iraqi population as a whole. Also, cytological abnormalities were not compared with histology results and data were based on opportunistic screening as opposed to an organized screening program.

\section{Conclusion}

The prevalence of abnormal cytology was high to that of hr-HPV testing, which could not allow the implementation of hr-HPV as a primary test in the national screening program in Iraq. Our data indicate that combined cytology and hr-HPV testing does not identify the same at-risk women and would entail higher diagnostic work-up, health-care costs. The high prevalence of abnormal cytology was enough to highlight the importance of the early detection of cervical cancers, thus saving lives. Further population-based prospective studies are needed to eliminate the drawbacks of our study and to determine nonhospital-based HPV prevalence in Iraqi women.

\section{Acknowledgments}

We think Ateaf Hamza, chief lad assistant who took part in this study. We also grateful to all patients involved in this study 


\section{Conflict of interest}

No conflict of interest.

\section{References}

1. Arbyn M, Weiderpass E, Bruni L, de Sanjosé S, Saraiya M, Ferlay J, et al. Estimates of incidence and mortality of cervical cancer in 2018: a worldwide analysis. Lancet Global Health. 2020;8(2):e191-e203.

2. Board IC. Annual report of cancer disease in Iraq 2013. In: Board C, editor: Ministry of Health; 2017

3. Ghittoni R, Accardi R, Chiocca S, Tommasino M. Role of human papillomaviruses in carcinogenesis. Ecancer Med Sci. 2015;9.

4. Papillomaviruses H. IARC monographs on the evaluation of carcinogenic risks to humans. Lyon, France: IARC. 2011.

5. Thun M, Linet MS, Cerhan JR, Haiman CA, Schottenfeld D. Cancer epidemiology and prevention: Oxford University Press; 2017.

6. de Kok IM, van der Aa MA, van Ballegooijen M, Siesling S, Karim-Kos HE, van Kemenade FJ, et al. Trends in cervical cancer in the Netherlands until 2007: has the bottom been reached? Int J Cancer. 2011;128(9):2174-81

7. Arbyn M, Ronco G, Anttila A, Meijer CJ, Poljak M, Ogilvie G, et al. Evidence regarding human papillomavirus testing in secondary prevention of cervical cancer. Vaccine. 2012;30:F88-F99.

8. Ronco G, Dillner J, Elfström KM, Tunesi S, Snijders PJ, Arbyn M, et al. Efficacy of HPV-based screening for prevention of invasive cervical cancer: Follow-up of four European randomised controlled trials. Lancet. 2014;383(9916):524-32.

9. Ogilvie GS, van Niekerk D, Krajden M, Smith LW, Cook D, Gondara L, et al. Effect of screening with primary cervical HPV testing vs cytology testing on high-grade cervical intraepithelial neoplasia at 48 months: The HPV FOCAL randomized clinical trial. JAMA. 2018;320(1):43-52.

10. Smith RA, Andrews KS, Brooks D, Fedewa SA, Manassaram-Baptiste D, Saslow D, et al. Cancer screening in the United States, 2019: A review of current American Cancer Society guidelines and current issues in cancer screening. CA: A Cancer J Clin. 2019;69(3):184-210.

11. von Karsa L, Arbyn M, De Vuyst H, Dillner J, Dillner L, Franceschi S, et al. European guidelines for quality assurance in cervical cancer screening. Summary of the supplements on HPV screening and vaccination. Papillomavirus Res. 2015:1:22-31.

12. Wright JrTC, Schiffman M. Adding a test for human papillomavirus DNA to cervical-cancer screening. New Engl J Med. 2003;348(6):489-90.

13. Curry SJ, Krist AH, Owens DK, Barry MJ, Caughey AB, Davidson KW, et al. Screening for cervical cancer: US Preventive Services Task Force recommendation statement. JAMA. 2018;320(7):674-86.

14. Elfström KM, Arnheim-Dahlström L, von Karsa L, Dillner J. Cervical cancer screening in Europe: quality assurance and organisation of programmes. Eur J Cancer. 2015;51(8):950-68.

15. Solomon D, Davey D, Kurman R, Moriarty A, O'Connor D, Prey M, et al. The 2001 Bethesda System: terminology for reporting results of cervical cytology. JAMA. 2002;287(16):2114-9.

16. Suteu O, Blaga ML, Nygård M, Leinonen MK, Nicula F, Pais R, et al. Prevalence of positive screening test results and agreement between cytology and human papillomavirus testing in primary cervical cancer screening in North-Western Romania. Eur J Cancer Prev. 2020;29(2):141-8.
17. Çalişkan E, CoŞkun SK, ÖztÜrk CE, Cangür Ş, Önal B. Analysis of HPV genotypes and liquid-based cervical cytology: Results from a tertiary academic center in northwestern Turkey. Jpn J Infect Dis. 2020:JJID. 2020.072.

18. Tahir QA, Bukhari MH. Evaluation of pre-malignant cervical lesions in females presenting with abnormal pelvic complaints. JPMA J Pak Med Assoc. 2020;70(2):272-5.

19. Nayak PK, Mitra S, Agrawal S, Hussain N, Thakur P, Mishra B. Role of various screening techniques in detecting preinvasive lesions of the cervix among symptomatic women and women having unhealthy cervix. 2020.

20. Gravitt PE, Paul P, Katki HA, Vendantham H, Ramakrishna G, Sudula M, et al. Effectiveness of VIA, Pap, and HPV DNA testing in a cervical cancer screening program in a peri-urban community in Andhra Pradesh, India. PLoS One. 2010:5(10):e13711.

21. Kim M-J, Kim JJ, Kim S. Type-specific prevalence of high-risk human papillomavirus by cervical cytology and age: Data from the health checkups of 7,014 Korean women. Obstet Gynecol Science. 2013;56(2):110-20.

22. Castle PE, Fetterman B. Five-year experience of human papillomavirus DNA and Papanicolaou test cotesting. Obstet Gynecol. 2009;113(3):595.

23. Wright Jr TC, Stoler MH, Behrens CM, Apple R, Derion T, Wright TL. The ATHENA human papillomavirus study: design, methods, and baseline results. Am J Obstet Gynecol. 2012;206(1):46. e1-. e11.

24. Vardell E. Global health observatory data repository. Med Ref Serv Quart. 2020;39(1):67-74

25. Monsonego J, Cox JT, Behrens C, Sandri M, Franco EL, Yap P-S, et al. Prevalence of high-risk human papilloma virus genotypes and associated risk of cervical precancerous lesions in a large US screening population: data from the ATHENA trial. Gynecol Oncol. 2015:137(1):47-54.

26. Kitchener H. HPV primary cervical screening: time for a change. 2015.

27. Bruni L, Diaz M, Castellsagué M, Ferrer E, Bosch FX, de Sanjosé S. Cervical human papillomavirus prevalence in 5 continents: meta-analysis of 1 million women with normal cytological findings. J Infect Dis. 2010;202(12):1789-99.

28. Forman D, de Martel C, Lacey CJ, Soerjomataram I, Lortet-Tieulent J, Bruni L, et al. Global burden of human papillomavirus and related diseases. Vaccine. 2012;30:F12-F23.

29. Tachezy R, Smahelova J, Kaspirkova J, Salakova M. Human papillomavirus type-specific prevalence in the cervical cancer screening population of Czech women. PLoS One. 2013:8(11):e79156.

30. Bao Y-P, Li N, Smith J, Qiao Y-L. Human papillomavirus type distribution in women from Asia: a meta-analysis. Int J Gynecol Cancer. 2008;18(1).

31. Ciotti M, Sesti F, Paba P, Benedetto A, Patrizi L, Criscuolo A, et al. Human papillomavirus (HPV) testing in the management of women with abnormal Pap smears. Experience of a colposcopy referral clinic. Eur J Gynaecol Oncol. 2004:25(5):577-84.

32. Gonzalez-Bosquet E, Almagro MM, Mora I, Suñol M, Callejo J, Lailla J. Prevalence of human papilloma virus infection of the uterine cervix in women with abnormal cervical cytology. Eur J Gynaecol Oncol. 2006;27(2):135-8

33. Perkins RB, Guido RS, Castle PE, Chelmow D, Einstein MH, Garcia F, et al. 2019 ASCCP Risk-Based Management Consensus Guidelines for abnormal cervical cancer screening tests and cancer precursors. J Lower Genital Tract Dis. 2020;24(2):102

34. Wright Jr TC, Massad LS, Dunton CJ, Spitzer M, Wilkinson EJ, Solomon D. 2006 consensus guidelines for the management of women with abnormal cervical cancer screening tests. Am J Obstet Gynecol. 2007;197(4):346-55.

This work is licensed under a Creative Commons Attribution-NonCommercial 3.0 Unported License which allows users to read, copy, distribute and make derivative works for non-commercial purposes from the material, as long as the author of the original work is cited properly. 